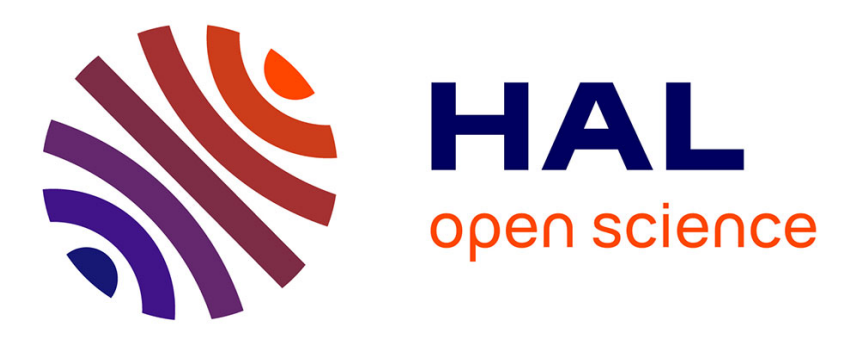

\title{
Auralisation dans les maquettes : traitement des réponses impulsionnelles
}

\author{
X. Meynial, V. Grillon, J. Polack, G. Dodd
}

\section{To cite this version:}

X. Meynial, V. Grillon, J. Polack, G. Dodd. Auralisation dans les maquettes: traitement des réponses impulsionnelles. Journal de Physique IV Proceedings, 1994, 04 (C5), pp.C5-135-C5-138. 10.1051/jp4:1994523 . jpa-00252916

\section{HAL Id: jpa-00252916 https://hal.science/jpa-00252916}

Submitted on 1 Jan 1994

HAL is a multi-disciplinary open access archive for the deposit and dissemination of scientific research documents, whether they are published or not. The documents may come from teaching and research institutions in France or abroad, or from public or private research centers.
L'archive ouverte pluridisciplinaire HAL, est destinée au dépôt et à la diffusion de documents scientifiques de niveau recherche, publiés ou non, émanant des établissements d'enseignement et de recherche français ou étrangers, des laboratoires publics ou privés. 


\title{
Auralisation dans les maquettes : traitement des réponses impulsionnelles
}

\author{
X. MEYNIAL, V. GRILLON, J.D. POLACK* et G. DODD**
}

\author{
Laboratoire d'Acoustique, URA 1011, Faculté des Sciences, Université du Maine, Avenue Olivier \\ Messiaen, BP. 525, 72000 Le Mans, France \\ * Acoustics Laboratory, Technical University of Denmark, Building 352, 2800 Lyngby, Denmark \\ ** Acoustics Research Centre, School of Architecture, University of Auckland, Private Bag, \\ 92019 Auckland, New Zealand
}

\begin{abstract}
Acoustical measurements using scale models offer a time and cost efficient method for determining the acoustical properties of enclosures. In order to listen in to the models, measured impulse responses have to be compensated for excessive air attenuation in the model, equalized for the transducers response, and their dynamic range has to be extended. The present paper describes corresponding processing, restricted to monaural signals.
\end{abstract}

Résumé: La mesure acoustique sur maquette procure une évaluation rapide et bon marché des propriétés acoustiques des espaces clos. De façon à pouvoir "écouter dans la maquette", les réponses impulsionnelles doivent être compensées de l'atténuation de propagation excessive dans la maquette, leur dynamique doit être étendue, et la réponse des transducteurs égalisée. Ce processus, restreint aux seuls signaux monauraux, est décrit dans cet article.

\section{O INTRODUCTION}

En acoustique architecturale, le but ultime dans la conception d'une salle est de satisfaire les auditeurs. L'approche traditionnelle pour y parvenir a été focalisée sur l'obtention de critères objectifs corrélés aux differents aspects de cette satisfaction.

Cependant, bien que de nombreuses études aient été effectuées sur la validité de ces critères objectifs [1], notre expérience nous a montré qu'ils ne reflètent pas pleinement limpression des auditeurs. Ainsi, de même que l'auralisation est de plus en plus utilisée en simulation numérique comme complément à la visualisation, il est également souhaitable d'obtenir une simulation sonore (auralisation) de la maquette. Cet article, qui se restreint à des signaux monauraux, décrit une approche préliminaire dans cette direction.

\section{MESURE DE REPONSES IMPULSIONNELLES SUR MAQUETTES}

La mesure sur maquette repose sur les lois de la similarité acoustique: le son se propage de la même manière dans une salle à pleine échelle que dans sa maquette, à ceci près que la fréquence est transposée par un facteur égal au facteur d'échelle. Ainsi, $1 \mathrm{kHz}$ à pleine échelle est équivalent à 50 $\mathrm{kHz}$ dans une maquette au 1:50ème. Cette transposition fréquentielle impose de grandes contraintes sur l'équipement de mesure : des cartes d'acquisition rapide $(1 \mathrm{MHz})$ doivent être utilisées et des transducteurs à très large bande sont nécessaires [2]. Le meilleur choix actuel est un microphone huitième de pouce à condensateur sensible jusquà $160 \mathrm{kHz}$ (seulement). A de telles fréquences, deux sortes de sources sonores sont disponibles : les sources à étincelle qui genèrent des impulsions courtes 
sous une distribution quasi omnidirectionnelle, et les transducteurs à film piezopolymer (PVDF) généralement utilisés pour les mesures pseudo-impulsionnelles (MLS). Cependant, les Réponses Impulsionnelles (RI) doivent être compensées autant que possible de la coloration des transducteurs. En plus de la limitation liée au Rapport Signal sur Bruit (RSB) et de la réponse du système de mesure, un biais majeur est apporté par l'atténuation exagérée du son se propageant dans l'air aux hautes fréquences. Nous décrivons dans ce qui suit une méthode pour compenser ces différentes limitations.

\section{TRAITEMENT DES REPONSES IMPULSIONNELLES}

Trois traitements doivent être appliqués aux RI mesurées.

- la compensation de l'atténuation de propagation sonore dans l'air

- l'extension de la décroissance au-delà du bruit de fond

- l'égalisation des transducteurs

\subsection{Compensation de l'atténuation sonore aérienne}

Les lois gouvernant l'atténuation du son dans l'air sont bien connues. De nombreuses études ont montré que cette atténuation peut s'exprimer au moyen d'un coefficient d'atténuation complexe $\alpha$ [3], coefficient qui croit rapidement aux hautes fréquences, mais aussi à chaque fréquence avec la température et l'humidité. De manière à éviter les inconvénients liés à l'utilisation de chambres spécifiques de tests ( coût, accessibilité à la maquette, lenteur), une compensation numérique peut être appliquée, qui consiste à retirer l'atténuation liée à la maquette puis à ajouter celle liée à la salle, référencée à des conditions atmosphériques standards de sorte qu'une normalisation est dans le même temps effectuée. Comme l'atténuation dans l'air dépend à la fois de la fréquence et de la distance parcourue par le son, cette distance étant directement liée au temps $\tau$ écoulé depuis son émission $(\mathrm{d}=c \tau)$, le processus de compensation peut être considéé comme un filtre variant dans le temps dont le gain logarithmique croit lineairement avec le temps comme on peut le voir sur la figure 1. Ce filtre est acausal et la longueur de sa reponse impulsionnelle augmente au cours du temps.

Un traitement rapide et précis peut être obtenu à l'aide d'une simple transformée de Fourier à court terme (TFCT). L'incrément de fenêtre doit être tel que la variation de la compensation entre deux fenêtres consécutives ne soit pas trop grande (typiquement $1 \mathrm{~dB}$ en amplitude et $10^{\circ}$ en phase dans le pire des cas, c'est à dire aux plus hautes frequences). Pour tenir compte de la nature acausale du filtre lors du filtrage FFT, un remplissage à zéro en début de fenêtre a été effectué en plus du classique remplissage en fin de fenêtre. Finalement, l'utilisation de la méthode "overlapp-add" assure un lissage de l'évolution des coefficients du filtre au cours du temps.

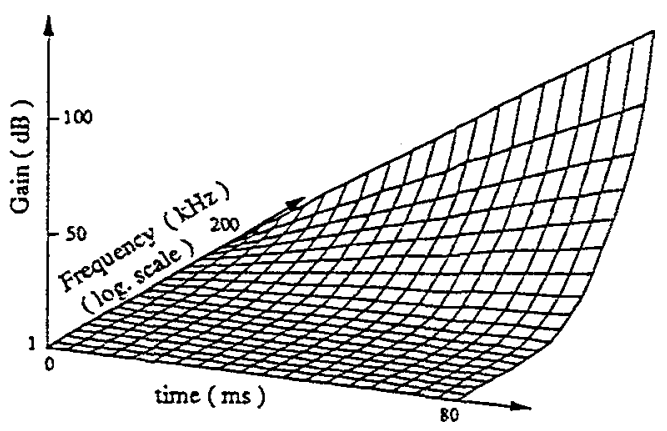

Fig. 1 : Evolution du gain du filtre compensant l'atténuation de propagation dans l'air dans le cas d'une maquette au 1:25 ème et pour des conditions atmosphériques usuelles.

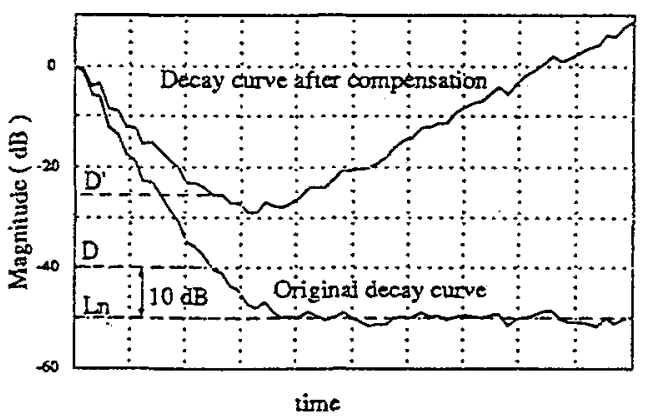

Fig. 2 : effet de la compensation de l'atténuation de l'air à $100 \mathrm{kHz}$ sur la courbe de décroissance $L n$ est le niveau de $B d F, D$ et $D^{\prime}$ les dynamiques avant et après compensation. 
La croissance linéaire du gain de cette compensation a cependant pour conséquence de relever considérablement le bruit de fond de la fin de la RI aux hautes fréquences comme le montre la figure 2. Un traitement doit donc être appliqué pour remédier à ce problème.

\subsection{Extension de la décroissance au-delà du bruit de fond}

Le bruit de fond affecte principalement la fin de la $\mathrm{RI}$ où il devient prédominant. Dans cette partie, qui correspond à la réverbération, la réponse de la salle peut être considérée sous un angle statistique. Donc, à partir du moment où le RSB dans une bande de fréquence donnée est suffisant pour fournir des informations valides sur le taux de décroissance dans cette bande, la partie tardive de la réponse peut être remplacée par un bruit d'amplitude décroissante, en supposant une décroissance exponentielle et une distribution de phase aléatoire. Ceci est souvent le cas excepté pour les fréquences en dehors de la bande passante des transducteurs. Un filtre passe-bande approprie est alors applique.

Pour tenir compte de la dépendance à la fois fréquentielle et temporelle du bruit de fond, un filtrage par TFCT a eté utilisé et une analyse par bandes critiques a été choisie car, en dehors de sa pertinence vis à vis du processus de perception humaine, elle procure un lissage approprié de la variance des courbes de décroissance dans chaque bande de fréquence. Ainsi, pour chaque bande, le plancher de bruit est déterminé et une pente de décroissance est extraite de la partie non contaminée par le bruit. Selon ces informations, une extrapolation convenable est effectuée qui prend en compte les differents problèmes posés par la reconstruction du signal [4]. En utilisant cette méthode, la décroissance peut être étendue aussi loin que l'on souhaite (fig.3) (dans la limite du bruit de calcul), correspondant éventuellement a une durée pouvant excéder la longueur d'acquisition originelle.

La robustesse de ce traitement est largement liée à l'évaluation de la décroissance, donc au RSB. Une erreur dans la pente de la décroissance induit l'émergence, dans la partie tardive de la RI, de la bande de fréquence correspondante. Le choix des bandes est donc très sensible et la courbe des pentes en fonction de la fréquence est lissée de manière à accroître la robustesse du traitement.

\subsection{Egalisation}

La réponse fréquentielle de la chaîne de mesure sur maquette (étincelle et micro 1/8") peut être appréciée sur le premier spectre (correspondant au son direct) de la figure 3. La nécessité d'une compensation est alors évidente. Cette compensation peut être fondée sur une mesure de référence en champ libre, évitant les diffractions sur les sièges qui affectent le son direct d'une RI mesurée dans une salle. Le but est d'obtenir un filtre stable realisant une égalisation en amplitude et en phase. Plusieurs techniques sont disponibles qui remplissent efficacement cette tâche [5]. 
Malheureusement, filtrer une $\mathrm{RI}$ complète de cette manière mène généralement à un résultat relativement déséquilibré. En effet, cette méthode suppose que la source soit répétable (entre la référence et la mesure des $\mathrm{RI}$ ), et qu'elle soit omnidirectionnelle (puisque tous les "rayons" émis subissent le même traitement), conditions qui ne sont jamais réalisées en pratique. Un compromis entre robustesse et précision du traitement doit alors être trouvé, et la reférence sonore doit être sélectionnée avec soin. Ceci montre encore une fois combien crucial est le contrôle des transducteurs pour la mesure acoustique.

\section{ECOUTE DES REPONSES IMPULSIONNELLES ET AURALISATION}

Ecouter les RI seules; avant toute convolution avec de la musique, est déjà très instructif. En effet, certains phénomènes comme les échos par exemple sont plus facilement détectables de cette manière. Cependant, une appréciation subjective plus naturelle sera obtenue par l'écoute de la convolution de cette RI par un signal anéchoïque musical ou de parole.

Les résultats sont très convaincants en ce qui concerne la compensation de l'atténuation sonore dans l'air où un très évident renforcement en hautes fréquences est constaté. Le traitement du bruit de fond est aussi satisfaisant : la décroissance apparait naturelle et aucune transition n'est perçue, même lorsque l'on écoute la RI seule - le cas le plus sensible. Les résultats concernant l'égalisation révèlent la grande sensibilité aux caractéristiques des transducteurs comme souligné au paragraphe 2.3, resultant parfois en un son très déséquilibré, spécialement en haute fréquence où le contrôle des transducteurs est le plus difficile. Globalement, ce problème apparaît comme étant le plus délicat.

Ajoutons que la limitation de bande passante $(6 \mathrm{kHz}$ au 1:25ème) est perceptivement très gênante. Un travail doit donc être fait pour étendre la réponse fréquentielle des $\mathrm{RI}$ mesurées dans les maquettes.

\section{CONCLUSION}

Cette étude préliminaire a montré qu'une auralisation de qualité sur maquettes en atmosphère ambiante est possible, rendant cette technique applicable à la conception acoustique d'espaces clos par les bureaux de consultation.

Des améliorations concernant la robustesse de certains traitements pourraient être fait. Les problèmes les plus délicats sont liés aux caractéristiques des transducteurs. La difficulté de contrôler leur directivité rend une égalisation précise problématique. La technologie actuelle des transducteurs spécialement celle des microphones- ne permet pas l'auralisation de musique avec une largeur de bande suffisante pour des maquettes d'echelle plus petite que le 1:25 ème. De manière à améliorer la qualité de l'auralisation sur les maquettes au 1:50 ème, la largeur de bande des réponses impulsionnelles mesurées doit être étendue.

\section{REFERENCES}

[1] L.Cremer, H.A.Müller, T.J.Shultz, "Principles and applications of room acoustics", (Applied Science, 1982)

[2] J.D.Polack, A.H.Marshall, G.Dodd, "Digital evaluation of the acoustics of small models : The MIDAS package", J. Acoust.Soc. Am. 85 (1989), 185-193

[3] J.D.Polack, S.Nezan, "Relaxation phenomena in viscothermal gases : application to attenuation and phase evaluation of sound waves", J. Acoust. 5(4) (Aug. 1992), 299-319

[4] J.D.Polack, X.Meynial, V.Grillon, "Auralization in scale models: processing of impulse responses", to be published in JAES.

[5] O.Warusfel, "Modélisation paramétrique des phénomènes acoustiques simples", Thèse de DocteurIngénieur, Université de Paris VI, 1985 